\title{
Le système éducatif de Madagascar
}

\section{Velomihanta Ranaivo}

\section{OpenEdition}

Journals

Édition électronique

URL : https://journals.openedition.org/ries/778

DOI : $10.4000 /$ ries. 778

ISSN : 2261-4265

\section{Éditeur}

France Education international

\section{Édition imprimée}

Date de publication : 1 décembre 2007

Pagination : 125-132

ISBN : 978-2-8542-0574-9

ISSN : 1254-4590

\section{Référence électronique}

Velomihanta Ranaivo, "Le système éducatif de Madagascar », Revue internationale d'éducation de Sèvres [En ligne], 46 | décembre 2007, mis en ligne le 30 juin 2011, consulté le 21 septembre 2021 URL : http://journals.openedition.org/ries/778 ; DOI : https://doi.org/10.4000/ries.778

Ce document a été généré automatiquement le 21 septembre 2021.

(c) Tous droits réservés 


\title{
Le système éducatif de Madagascar
}

\author{
Velomihanta Ranaivo
}

\section{Aperçu historique}

1 Décrire l'éducation à Madagascar, c'est évoquer les apports des influences extérieures qui ont considérablement marqué l'histoire de la Grande île, en particulier depuis le début du $\mathrm{XIX}^{\mathrm{e}}$ siècle. En effet, l'école est née avec l'arrivée des envoyés de la London Missionary Society dont l'œuvre civilisatrice était " conçue et organisée dans un but religieux », tout en appuyant le « développement de l'impérialisme britannique » au sein d'une monarchie soucieuse au départ d'assurer « l'ouverture de Madagascar au travail et au commerce » (Belrose-Huygues, 1993 : 189 et 191).

2 Après la loi d'annexion du 6 août 1896, l'institution scolaire a été réorganisée de façon à constituer un instrument de la domination coloniale française qui s'étendait à tous les secteurs d'activités politiques, économiques et socioculturelles.

3 L'indépendance acquise en 1960 a, d'une certaine façon, renforcé les séquelles d'une gestion des affaires orientée au profit d'une oligarchie (inter)nationale composée de dirigeants, d'industriels et de commerçants. La politique scolaire était alors calquée sur celle de la métropole: programme français, personnel formé à la française. Ce système élitiste a été totalement remis en question par les événements de 1972. Cependant, la révolution socialiste qui en était issue n'est pas réellement parvenue à tenir toutes ses promesses en matière de décentralisation et de malgachisation de l'enseignement.

4 Depuis 1991, "la transition démocratique ", l'ouverture au plurilinguisme et le choix affirmé très récemment en faveur de l'ultralibéralisme semblent animer une dynamique censée permettre l'instauration d'une éducation plus moderne, plus équitable et plus performante.

5 Ainsi, les caractéristiques du système éducatif malgache actuel découlent d'une «nouvelle donne » dans laquelle défis, options et contraintes s'inscrivent dans un projet de société marqué par la recherche du « développement rapide et durable $»^{1}$. Respectueux 
des «droits et devoirs (...) énoncés dans la Constitution » ainsi que de "certaines valeurs spécifiques (...) telles que les notions de "vie" (aina), "âme-raison constitutive de l'homme" (fanahy maha-olona), de "vertu, sainteté" (hasina) ou "amitié, bonnes relations" ( fihavanana), "fidèle aux engagements internationaux" ", l'État fait de l'éducation "une priorité nationale absolue » et définit l'enseignement et la formation malgaches comme des processus censés "préparer l'individu à une vie active intégrée dans le développement social, économique et culturel du pays $»^{2}$. Face à ces grands principes, tout est conçu pour soutenir les efforts (inter)nationaux en vue du redressement du secteur éducatif : le cadre institutionnel et administratif, les structures et les stratégies.

\section{Une nouvelle configuration administrative et territoriale}

6 Dans un pays où la tradition centralisatrice a été marquante, on note aujourd'hui une volonté affirmée de rompre avec les pratiques passées. L'un des changements d'envergure est la mise en œuvre du processus participatif par le biais de la création de vingt-deux régions (Faritra) en juin 2004³. Différents niveaux de prise de décision ont été définis qui devraient permettre dorénavant une meilleure mise en cohérence des actions, du sommet à la base :

- le président de la République «détermine et arrête, en conseil des ministres, la politique générale de l'État » (article 54 de la Constitution);

- le Parlement est doté de fonctions législatives spécifiques ;

- les ministères chargés de l'éducation, de l'enseignement et de la formation «mettent en œuvre (...) la politique nationale » dans ces domaines ;

- pour ce qui relève des actions du ministère de l'Éducation nationale et de la recherche scientifique (MENRS), vingt-deux directeurs régionaux sont nommés; sous leur tutelle fonctionnent cent onze chefs de circonscriptions scolaires secondés par des équipes d'encadrement et de suivi au plan local ; c'est de leur autorité que relèvent tous ceux qui assurent la gestion des établissements et des écoles grâce à la collaboration de différents partenaires tels que les Fikambanan'ny ray aman-drenin'ny mpianatra (FRAM : association des parents d'élèves), les ONG, etc.

7 À terme, dans le cadre du programme de "gouvernance de proximité », le développement éducatif « sera propulsé par les initiatives régionales et communales »"

\section{Structures et champs d'action}

En conformité avec le Document de stratégie de réduction de la pauvreté finalisé en juillet 2003 ainsi que le Plan d'action Madagascar 2007-2012 (MAP : Madagascar Action Plan), la Politique générale de l'État 2006 inscrit comme l'un de ses axes prioritaires «la sécurisation humaine et sociale ». À ce titre, plusieurs entités se partagent la tâche qui consiste à assurer à " tous les Malgaches une éducation de qualité », qu'elle soit formelle ou non ${ }^{5}$. Il s'agit entre autres du ministère de l'éducation nationale et de la recherche scientifique avec deux de ses missions principales, l'enseignement scolaire et la formation, l'enseignement supérieur et la recherche. 
Évolution des dépenses totales du MENRS par niveau d'enseignement de 2002 à 2004 (en milliards d'ariary*)

\begin{tabular}{|l|l|l|l|}
\hline Niveau d'enseignement & $\mathbf{2 0 0 2}$ & $\mathbf{2 0 0 3}$ & $\mathbf{2 0 0 4}$ \\
\hline Enseignement primaire & 64,61 & 118,92 & 77,78 \\
\hline Enseignement secondaire général & 29,68 & 49,97 & 81,69 \\
\hline Formation professionnelle et technique & 6,02 & 10,47 & 16,05 \\
\hline Enseignement supérieur & 34,53 & 15,00 & 7,66 \\
\hline
\end{tabular}

* 1 ariary $=5$ francs malgaches ou fmg ; 1 euro $=2500$ ariary soit $12500 \mathrm{fmg}$.

9 Selon les données disponibles relatives au financement de la réforme, les dépenses d'éducation représentaient $2,3 \%$ du PIB en 2001 contre 3,8\% en 2004. Les efforts se concentrent sur l'enseignement primaire, compte tenu de l'initiative en faveur de l'éducation pour tous (EPT) et de l'objectif global du Document de stratégie de réduction de la pauvreté .

Dans cette optique et pour plus d'efficacité, le primaire a été restructuré en deux niveaux :

- l'enseignement fondamental (EF1) correspond à une scolarité obligatoire de cinq années; l'enfant y entre à l'âge de six ans ;

- l'enseignement fondamental du deuxième cycle (EF2), d'une durée de quatre années.

11 Il est à noter que $94 \%$ des enfants âgés de six ans sont scolarisés dans l'EF1 contre $27 \%$ des jeunes âgés de onze à quatorze ans dans l'EF2.

12 Les actions engagées depuis 2003 font apparaître des avancées dans ce domaine :

- un taux net de scolarisation qui dépasse les prévisions, et qui s'explique par le double effet de « l'exonération des droits d'inscription et de la distribution de kits scolaires ${ }^{7}$;

- une augmentation du nombre d'enseignants, surtout ceux pris en charge par l'association de parents d'élèves ${ }^{8}$;

- un taux d'achèvement de $57 \%$ en 2006 ;

- un taux de réussite au CEPE de 64,6 \% en 2005 ;

- dans l'enseignement fondamental du deuxième cycle, le taux d'achèvement est de $14,7 \%$ en 2004 et le nombre d'enseignants est en hausse (soit 8901 pour 420000 élèves); le pourcentage d'admis au BEPC s'élève à 39,1\% en 2005 puis 46,1\% en 2006.

13 Quant à l'enseignement secondaire qui s'étale sur trois ans et dont le taux d'achèvement de $6 \%$ coïncide avec les prévisions pour les années 2003-2004, une tendance à la hausse est observée en ce qui concerne le nombre d'élèves $(89000)$; le taux de réussite au baccalauréat avoisine les $40 \%$ (37,2 \% en 2002, 33,3 \% en 2004 contre $41 \%$ en 2006).

14 Ces phénomènes globalement positifs au plan des chiffres, et surtout dans l'enseignement fondamental, ne doivent pas masquer un degré de performance qui demeure faible : un pourcentage important de redoublants (30\% en EF1 pour l'année 2004), une légère 
atténuation en EF2 (14,4\%), dans l'enseignement secondaire (14,5\%) et dans l'enseignement supérieur (18,2\%). D'autres indices restent préoccupants :

- le petit nombre d'élèves qui entrent au primaire sans avoir pu bénéficier d'un passage dans un centre préscolaire (un enfant sur vingt) ;

- un nombre réduit d'élèves ayant accès à l'enseignement secondaire général ( $9 \%$ seulement de ceux qui se situent dans la tranche d'âge quinze/dix-huit ans sont inscrits à ce niveau) ;

- le nombre restreint de diplômés de l'enseignement supérieur (4 750 en 2006);

- le taux d'abandon à presque tous les niveaux du primaire $(24,5 \%$ en première année entre 2004-2005 et $2005-2006 ; 18,5 \%$ en quatrième année pour la même période) jusqu'à l'enseignement supérieur ( $24,2 \%$ en $2004 ; 32,4 \%$ en 2005).

Des stratégies de redynamisation sont en cours afin d'améliorer ces résultats. Elles portent sur la gestion du système lui-même et sur la prise de mesures administratives et pédagogiques de toutes sortes :

- poursuite du programme de constructions scolaires pour l'EF1 (1 860 en 2006), parallèlement au recrutement d'enseignants, en priorité ceux qui relèvent de l'association des parents d'élèves (2 300 postes en moyenne par an sur dix ans) ;

- « objectif zéro redoublement » maintenu ;

- réforme prévue concernant le système d'évaluation ;

- formation à la gestion des classes multigrades (elles représentent $60 \%$ des classes malgaches), formation à la pédagogie des grands groupes qui est généralisée dans l'enseignement primaire (pour un ratio élèves/maître de 52 en 2005-2006); mise en application de l'approche par compétences qui constitue le principal moteur de la réforme éducative à ce niveau du système (vingt mille enseignants formés au cours élémentaire en 2006).

16 En effet, si l'on en croit le MAP, la formation est au cœur des programmes prioritaires du gouvernement. Après les expériences des années quatre-vingts (recrutement en masse au niveau $\mathrm{BEPC}$, trois mois de formation à contenu essentiellement idéologique), une autre hypothèse a été retenue avec une alternance plus construite entre théorie et pratique (actuellement, six mois de formation intra muros et six mois de stage en responsabilité). L'Institut national de formation pédagogique créé en 1996 assure ce volet des opérations grâce au concours des dix-huit centres régionaux qui lui sont rattachés. Quant au dispositif d'encadrement, il s'appuie sur les conseillers pédagogiques (une année de cours théorique; une année d'application pratique) et les inspecteurs (même schéma de formation).

Dans l'enseignement fondamental du deuxième cycle (formation des enseignants à l'INFP pendant deux ans avec un stage de cinq mois), le mouvement d'expansion des collèges est encouragé avec l'appui du privé. Afin de rendre opérationnels les élèves qui choisissent de rejoindre le marché du travail à la fin de ce niveau d'études, le programme est soumis à une réflexion qui touche le socle de compétences et le renforcement de l'anglais dans un contexte de mondialisation incontournable.

la même façon, l'enseignement secondaire est appelé à voir ses curricula réaménagés en fonction des besoins économiques et de la préparation à l'enseignement supérieur (formation des élèves-professeurs de lycées à l'ENS pendant cinq ans après le baccalauréat et recrutement sur concours).

19 L'entrée dans la culture technologique est en cours de concrétisation par le programme de mise en place de centres de ressources dans les lycées. 

du cycle du primaire à sept ans. Le schéma $5+4+3$ est donc voué à se transformer en formule $7+3+2$, de manière à « augmenter le nombre moyen d'années de scolarité et à améliorer le niveau éducatif de la population $»^{10}$.

La démarche qualité s'étend également à l'enseignement technique et à la formation professionnelle dont les offres et les dispositifs sont amenés à se diversifier et à s'adapter aux réalités régionales dans le cadre du défi de la décentralisation (aujourd'hui, 350 jeunes en formation professionnelle pour 100000 habitants).

tratégie de réduction de la paurreté et le Plan daction Madagascar 2007-2012 concluent à la nécessité d'un redéploiement des initiatives en ce qui concerne l'enseignement supérieur. Deux directions sont envisagées à court et moyen terme, à savoir l'adoption d'une "nouvelle culture académique " grâce au basculement vers le système licence-mastère-doctorat (LMD) et la redéfinition des modalités de gestion et de financement de la formation et de la recherche par des «mécanismes d'assurance qualité interne des programmes ». La part du privé et de l'e-learning sont au cœur de la politique de relance dans ce sous-secteur, si l'on en croit les schémas prévisionnels établis jusqu'en 2015-2016: un objectif de 7006 étudiants en 2007-2008 en formation à distance au Centre national de télé-enseignement de Madagascar (CNTEMAD) ${ }^{11}$ contre 8500 en 2015-2016; dans le même temps, les institutions privées prendront en charge 7774 étudiants en 2007-2008 contre 15126 en 2015-2016. Des réflexions sont initiées dans les universités au sein du comité de pilotage LMD et des comités préparatoires LMD par établissement à l'Université d'Antananarivo.

Finalisées ou non, ces multiples interventions contribuent à la "transformation de l'éducation ", l'un des huit engagements du Plan d'action Madagascar 2007-2012. Elles se réfèrent à un changement non seulement de structures mais aussi de philosophie et de paradigme dont il convient de préciser quelques éléments moteurs face aux «nouveaux chantiers de la réforme $»^{12}$.

24 C'est sur cette toile de fond que viennent prendre place et signification les débats sur l'éducation.

\section{Questions vives}

\section{La décentralisation en marche}

Dans l'histoire du champ éducatif malgache, la décentralisation a déjà fait l'objet d'une réforme engagée au temps de la II ${ }^{e}$ République (cf. Loi d'orientation $n^{\circ} 78040$ article 8$)^{13}$.

Alors qu'à cette époque les écoles étaient « dans leur essence des instruments au service de l'édification du socialisme à Madagascar » et devaient, en tant que telles, se conformer en tout point aux mots d'ordre du «Pouvoir révolutionnaire $»^{14}$, l'approche est ici totalement différente en ce qu'elle s'appuie sur le "principe de subsidiarité et de complémentarité des compétences avec la participation active des forces vives locales ", à tous les niveaux du découpage administratif (Faritany, Distrika, Kaominina, Fokontany) ${ }^{15}$.

Dans le cadre de cette réforme tendant à mettre en place "une société de bonne gouvernance ", la capacité d'innovation et d'adaptation requise des acteurs passe par «le renforcement de capacités managériales, de gestion, de pilotage ${ }^{16}$, ce qui ne va pas sans poser problème : «insuffisance de volonté politique », « conflits de pouvoir » sans parler 
des obstacles financiers, matériels et culturels tels que la "résistance au changement »; ce sont du moins les risques déjà identifiés par les responsables ${ }^{17}$.

\section{Partenariat public/privé : quelles articulations fonctionnelles ?}

La gratuité figurait en bonne place dans les visées politiques, éthiques et économiques du régime socialiste, comme le confirme l'article 51: "conformément au Livre rouge, l'objectif final est l'instauration d'un système d'enseignement gratuit pour tous, à tous les niveaux, et dont la charge sera supportée par la nation toute entière ». Et d'ajouter : "L'existence de système d'écoles payantes ne se justifie que dans la phase transitoire d'acheminement vers un système scolaire gratuit à tous les niveaux ». Cette prise en charge suffisait-elle à justifier le contrôle sévère exercé sur l'enseignement privé ${ }^{18}$ Avec l'ouverture du pays au pluralisme politique et culturel et le mouvement de "désidéologisation" qui la traduisait au plan des textes de référence, c'est la contractualisation de l'approche qui a commencé à prédominer à partir de 1995; il s'agissait alors d'aider à la libération des initiatives (cf. Loi d'orientation $n^{\circ} 94033$ du 13 mars 1995, article 11). Une instance intégrée dans l'organigramme du ministère a été créée pour gérer la problématique des interventions du privé: l'office national de l'enseignement privé.

Aujourd'hui, l'état d'esprit a évolué vers la systématisation de la démarche partenariale définie comme « règle dans l'exécution de la politique d'éducation et de formation » (Loi d'orientation $n^{\circ} 200404$ du 26 juillet 2004, article 11). De plus, on note une clarification des missions, des droits et devoirs des établissements d'enseignement privé.

Dans le contexte qui prévaut, la tendance semble à l'acceptation d'un partage de responsabilités avec le public, dans le cadre d'intérêts bien compris ${ }^{19}$. Une des tendances émergentes pourrait même être l'extension du partenariat dans l'enseignement supérieur ${ }^{20}$. Or cela suppose une concertation à la base, et sur des points importants qui structurent l'acte d'éduquer et de former.

\section{Politique linguistique et politique de formation des ressources humaines}

31 Parmi les sujets qui alimentent la demande sociale et qui agitent périodiquement le monde de l'éducation à Madagascar, on peut citer celui des langues et des compétences de leurs utilisateurs : dans le fond, c'est le même débat récurrent et la réflexion risque de tourner en rond longtemps si une démarche systémique, systématique et intégrée n'est pas envisagée.

32 Une brève incursion dans le passé permet d'attirer l'attention sur des expériences diamétralement opposées sur le terrain des langues. Passant brutalement et sans évaluation formelle d'un extrême à l'autre, élèves, enseignants, programmes et évaluations basculent de la malgachisation dans les années quatre-vingts à la gallicisation dans les années quatre-vingt-dix. Le fait de se doter de trois langues officielles depuis cette année ${ }^{21}$, lors du référendum d'avril 2007, ne résout pas automatiquement les problèmes. Il y va d'abord des représentations des locuteurs. À ce sujet, des recherches récentes soulignent le fait que, pour une très grande majorité de Malgaches, la communication se fait uniquement dans la langue nationale ${ }^{22}$. Il est question aussi et 
surtout de la stratégie déployée en vue d'apprendre et de faire apprendre grâce à l'utilisation de telle ou telle langue dans tel ou tel domaine d'intervention. Aussi l'harmonisation des parcours, tant sur l'axe vertical des niveaux (du primaire à l'enseignement supérieur) que sur l'axe horizontal des filières, exige-t-elle une réflexion de fond et d'ensemble. Un début d'action dans ce sens a pu associer cette année des établissements que rapprochent des problématiques croisées de formation d'enseignants comme l'INFP, les facultés et l'ENS, afin d'éviter les risques liés à l'application d'une vision éclatée du système qui n'a que trop perduré.

\section{Quelle recherche pour quel développement?}

Un grand ministère gère les volets éducation et recherche scientifique. Cela peut être considéré comme un atout. Reste à préciser la nature de l'activité de recherche et les moyens mis à sa disposition. Les documents de référence officiels orientent clairement les priorités vers des programmes qui soient en réponse aux besoins nationaux: "développement des régions et filières de production », et cela sur la base de 0,05\% du budget en 2006 et $0,10 \%$ en $2012^{23}$. Des passerelles sont à établir entre universités et centres de recherche avec une participation du privé et des partenaires extérieurs. Certains souhaitent avant tout la reconnaissance du statut même des chercheurs qui risquent paradoxalement d'être des laissés pour compte du développement s'ils ne bénéficient pas d'appui conséquent.

La réforme que l'on vient d'évoquer dans ses grands axes en est à ses premiers frémissements. Certes la question des moyens est essentielle dans la recherche de l'efficacité interne et externe du système. C'est ainsi que grâce au Programme d'appui des Nations unies 2008-2011, le MAP bénéficie d'une contribution de trois cent millions de dollars USD. Cependant, au-delà des moyens, ce sont les modèles culturels qui déclenchent et soutiennent en permanence les comportements innovants ou bloquants. De nombreux chercheurs l'ont souligné. Il est donc urgent de (re)travailler cette dimension, d'autant que le cadre politique semble bien avoir renforcé sa légitimité par la voie des élections législatives du 23 septembre 2007 (106 sièges pour le parti présidentiel sur 127). Le développement du secteur et de la nation toute entière en dépend.

\section{Madagascar}

Superficie : $592000 \mathrm{~km}^{2}$

Capitale : Antananarivo

Population (estimation 2003) : 16600000 habitants

Pourcentage de jeunes : 56,6 \% ont moins de 20 ans, la majorité en milieu rural

Monnaie : Ariary

Économie : $82 \%$ des actifs occupés dans le secteur primaire

Langues officielles : malgache, anglais et français

Nature de l'État : République

Budget éducation \% PIB : 3, $8 \%$ en 2006

Taux d'alphabétisme adolescents/adultes de plus de 15 ans : $52 \%$

Taux d'achèvement de l'éducation primaire : 57 \% en 2006

Taux d'achèvement dans l'enseignement secondaire général : 7 \% en 2006 


\section{NOTES}

1. Le développement durable est un mode de développement «qui répond aux besoins des générations présentes sans compromettre ceux des générations futures » (Rapport Brundtland, 1987 cité dans le Programme national de décentralisation et de déconcentration, PN2D, Lexique en 2D, document émanant de la Présidence de la République en collaboration avec le ministère de la décentralisation et de l'aménagement du territoire ainsi que le ministère de l'intérieur et de la réforme administrative, version du 16 octobre 2006).

2. Loi $n^{\circ} 2004-04 \mathrm{du} 26$ juillet 2004 portant orientation générale du système d'éducation, d'enseignement et de formation à Madagascar.

3. Ce sont « des collectivités publiques à vocation essentiellement économique et sociale (...) Elles assurent (...) la planification, l'aménagement du territoire et la mise en œuvre de toutes les actions de développement » (PN2D, Lexique en 2D).

4. PN2D, p. 13.

5. Loi d'orientation $n^{\circ} 2004-04$, art. 22.

6. Document de stratégie de réduction de la pauvreté dont la finalisation a débouché sur l'accès au premier lot de financement de l'initiative pour les pays pauvres très endettés (IPPTE) au cours des années 2001-2002.

7. MENRS : La réforme de l'éducation: point de situation et préparation des nouvelles orientations, février 2005, p. 6.

8. 47287 enseignants du public dont 17690 payés par le FRAM pour 3366600 élèves au total.

9. MENRS: La réforme de l'éducation: point de situation et préparation des nouvelles orientations, février 2005, p. 6.

10. Madagascar Action Plan, p. 53.

11. MENRS : La réforme de l'éducation: point de situation et préparation des nouvelles orientations, février 2005, p. 22.

12. Ibid., p. 25.

13. Loi du 17 juillet 1978 promulguée à l'époque de la République démocratique de Madagascar.

14. Loi d'orientation $\mathrm{n}^{\circ} 78040$, art. 52 et 53 .

15. PN2D, p. 13. Vision qui était en germe dans les documents initiaux de préparation aux élections présidentielles de 2001 tels que Vina rafi-piaraha-monina (Projet de société) du parti présidentiel Tiako i Madagasikara.

16. PN2D, p. 34 .

17. Ibid., p. 49-50.

18. Les modalités fixées en matière d'aide et de contrôle étaient là pour le rappeler: elles s'effectuaient « chaque année en fonction de la progression vers le but final »; l'aide dépendait aussi bien «des résultats obtenus aux différents examens officiels que du rapport des contrôleurs ».

19. Interview de B. Rajoelisona, directeur national de l'enseignement privé de Madagascar, « Les enseignements de l'enseignement », Revue de l'Océan Indien, n² 271, novembre 2006, p. 52.

20. S. Ramaromanana, "Et si on fait appel au privé... pour assurer la formation universitaire? ", Revue de l'Océan Indien, n² 271, novembre 2006, p. 54.

21. Le malgache langue maternelle et national, le français et l'anglais.

22. Z. Ramandazafy, «Le malgache, langue d'enseignement. Faire taire le cœur pour laisser parler la raison », Revue de l'Océan Indien, juillet-août 2004, pp. 62-64.

23. Madagascar Action Plan, p. 58. 
INDEX

Mots-clés : politique éducative, système éducatif, système scolaire

Index géographique : Madagascar

\section{AUTEUR}

\section{VELOMIHANTA RANAIVO}

Maître de conférences, Université d'Antananarivo, présidente du comité préparatoire LMD ENS, Madagascar. 